\title{
Low-rank Sparse Matrix Decomposition for Sparsity-driven SAR Image Reconstruction
}

\author{
Abdurrahim Soğanlı and Müjdat Çetin \\ Faculty of Engineering and Natural Sciences \\ Sabancı University \\ Orhanl1, Tuzla, 34956 Istanbul, Turkey \\ Email: \{soganli,mcetin\}@sabanciuniv.edu
}

\begin{abstract}
We consider the development of a synthetic aperture radar (SAR) image reconstruction method that decomposes the imaged field into a sparse and a low-rank component. Such a decomposition is of interest in image analysis tasks such as segmentation and background subtraction. Conventionally, such operations are performed after SAR image formation. However image formation methods may produce images that are not well suited for such interpretation tasks since they do not incorporate interpretation objectives to the SAR imaging problem. We exploit recent work on sparse and low-rank decomposition of matrices and incorporate such a decomposition into the process of SAR image formation. The outcome is a method that jointly reconstructs a SAR image and decomposes the formed image into its low-rank background and spatially sparse components. We demonstrate the effectiveness of the proposed method on both synthetic and real SAR images.
\end{abstract}

Keywords-Synthetic aperture radar (SAR), image reconstruction, low-rank sparse matrix decomposition

\section{INTRODUCTION}

Decomposing an image or signal into two or more parts has found use in many image analysis problems such as background subtraction, denoising, and segmentation [1]. These methods are used for supporting later stages of analysis such as object recognition. Therefore, the accuracy of the decomposition affects the performance of these inference or decision making tasks. Decomposing sparse objects and the background of the scene is one of the typical problems for the above mentioned applications. Recently, there has been interest in this decomposition problem, where the background of the scene is assumed to be a low-rank matrix. Besides, recent pieces of work have reported that under very mild conditions, decomposition of low-rank and sparse components (LRSD) from partial or corrupted measurements is possible [2]. The LRSD model has been used in many applications including face recognition [3] and background subtraction [4]. LRSD has also been used as a prior term in inverse problems in imaging, such as medical image reconstruction [5].

Synthetic aperture radar (SAR) imaging applications also involve the above mentioned interpretation tasks such as object recognition and object tracking. These applications usually require high resolution images for accurate decision or interpretation. Thus, image formation of SAR data comes into prominence. In general, SAR image formation and further

This work has in part been supported by a Graduate Fellowship from the Scientific and Technological Research Council of Turkey (TUBITAK). image analysis are performed in a decoupled fashion. Interpretation methods are applied to the SAR image after SAR image formation has been completed. Current SAR systems achieve image formation by using Fourier transformationbased algorithms [6], [7]. These purely data-driven conventional image formation algorithms are simple and efficient. However, they suffer from noise, speckle, limited-resolution, and sidelobe artifacts due to the limited bandwidth of the SAR systems. Such artifacts and limitations pose challenges for further interpretation tasks.

Recent years have witnessed the incorporation of sparsity as prior information into the SAR image formation problem to cope with the shortcomings of the conventional methods. These sparsity-driven methods [8], [9] assume that the underlying reflectivity field admits sparsity in a particular domain and have been shown to offer better reconstruction quality as compared to conventional methods. In particular, analysisbased models, such as in [8] enhance point-based and regionbased structures by imposing sparsity on the features of the reflectivity, whereas synthesis-based models [9] represent the reflectivity field sparsely with a dictionary by imposing sparsity on the coefficients of representation through a dictionary. However both models only enhance predefined features of the reflectivity field hence they may suppress non-smooth regions and patterns involved in the scene which are useful for further analysis tasks and can be represented as low-rank structures. Thus, these methods may also produce an unsuitable image for interpretation methods since the higher level objectives of the interpretation stage are not used in the formation process.

A SAR image that is potentially better suited to the higherlevel inference goals might be formed by adding information about these objectives into the SAR imaging problem. In this work, we integrate the LRSD framework into the SAR imaging problem. The proposed method has two advantages. Firstly, we decompose sparse components and low-rank background in the SAR scene while reconstructing the SAR image. Therefore, for SAR applications, the proposed method provides two additional images along with a composite SAR image: a sparse image which contains sparse objects in the scene and the low-rank background image. Secondly, the proposed method may essentially perform partial image analysis during the reconstruction phase, if for example the application involves segmentation of the sparse objects, or subtraction of the background from the SAR scene. 


\section{PRELIMINARIES}

In this section we present the mathematical observation model for spotlight-mode SAR and review recent developments on the decomposition of matrices into their sparse and low-rank components.

\section{A. SAR Observation Model}

The SAR imaging problem is an inverse problem in which the reflectivity field is to be reconstructed from backscattered observations. SAR collects the returned signals from this field as it traverses its flight path. In spotlight-mode SAR, return signals for every aperture position are collected by a radar sensor which is continuously steered to the ground patch. After some pre-processing steps, the relationship between the return signals for a given angle $\theta$ and the complex-valued reflectivity field $f(x, y)$ becomes:

$$
r_{\theta}(t)=\iint_{x^{2}+y^{2} \leq r^{2}} f(x, y) e^{-j K(t)(x \cos \theta+y \sin \theta)} d x d y
$$

where $r$ is the radius of the reflectivity field, and $K(t)$ is the radial spatial frequency. These return signals are called phase histories and each return corresponds to a limited slice from the 2D Fourier transform of the reflectivity field $f(x, y)$ at angle $\theta$. We can stack all phase history samples and reflectivity field samples to obtain the following model:

$$
g=H f+n
$$

where $H$ represents the observation model in (1) and $n$ is measurement noise. This inverse problem is ill-posed since both the bandwidth of the SAR system and aperture positions are limited.

\section{B. Low-rank Sparse Decomposition (LRSD)}

Decomposing a matrix $A$ into its low rank $D$ and sparse $S$ components has been of interest in recent years. For the imaging problem, this can be considered as decomposing an image into the low-rank background and sparse part. Sparse part may consist of the dominant point objects in the image. In particular low-rank sparse matrix decomposition can be expressed as:

$$
\min _{D, S} \operatorname{rank}(D)+\lambda\|S\|_{0} \quad \text { s.t. } \quad A=D+S
$$

where $\lambda$ balances the two constraints. Note that the rank minimization problem is NP-hard and involves minimizing the number of nonzero singular values. Recent pieces of work [10], [11] however have proved that the nuclear norm, defined as

$$
\|F\|_{*}=\sum_{i=1}^{r} \sigma_{i}(F)
$$

can, under certain conditions, be used as a surrogate convex form of the rank minimization constraint. After this relaxation, the resulting convex optimization problem of LRSD is given by

$$
\min _{D, S}\|D\|_{*}+\lambda\|S\|_{1} \quad \text { s.t. } \quad A=D+S
$$

Several efficient solutions have been proposed for the Augmented Lagrangian form of the problem using gradient descent algorithms [12], [13]. This framework has recently found use in image processing applications and some preliminary ideas have been reported for SAR imaging as well [14], [15].

\section{PROPOSED METHOD}

In order to exploit the LRSD framework in SAR image reconstruction, the vectorized form of the SAR image $f \in \mathbb{C}^{N}$ should be converted to the matrix form. The image form of this vector with size $\sqrt{N} \times \sqrt{N}$ can be used as a matrix. However, low-rank assumption of a complete SAR image is unrealistic and may provide inefficient results. Therefore, we use a patchbased method for constructing the matrix form of the SAR image. Let $R$ be the linear operator that constructs a patchbased matrix from the image. Image patches $f_{i} \in \mathbb{C} \sqrt{n} \times \sqrt{n}$ are obtained from the image by using a sliding window starting from top-left of the image to the bottom-right. Note that, for the sake of simplicity and with a slight abuse of notation, we use $f_{i}$ for both the matrix and the vectorized forms of the image patches. Vectorized form of these patches form columns of the patch-based matrix. The patch-based matrix $F \in \mathbb{C}^{n \times K}$ has the form of

$$
F=\left[\begin{array}{cccccc}
\mid & \mid & & & & \mid \\
f_{1} & f_{2} & \cdot & \cdot & \cdot & f_{K} \\
\mid & \mid & & & & \mid
\end{array}\right]
$$

where $K$ depends on the sliding distance and the size of the patches. This matrix can be deconstructed to recover the original image. Note that if the sliding distance is smaller than $\sqrt{n}$, there will be overlapping patches and $F$ will contain repeated entries for the same pixels. We take mean of these values in the process of deconstructing $F$ to reconstruct the image $f$. This patch-based method has been used in [16] for small target detection. SAR reflectivities, which are complexvalued, usually exhibit random phase, which should be taken into account in imposing sparsity and low-rank structure to the complex-valued SAR scene. Thus, we represent the magnitude of the SAR image with this matrix $F$ such that $F=R(|f|)$. The reconstruction process can be expressed as $|f|=R^{*}(F)$. Let $B$ and $S$ be the patch-based image for the low-rank background and the sparse part, respectively. Thus, the SAR observation model can be expressed as

$$
g=H \Theta R^{*}(B+S)+n
$$

where the diagonal matrix $\Theta$ contains the exponentiated phases of the reflectivity field. Using this notation, the proposed LRSD-based SAR imaging problem can be expressed as follows:

$$
\begin{aligned}
\widehat{B}, \widehat{S}, \widehat{\Theta}=\arg \min _{B, S, \Theta}\left\|g-H \Theta R^{*}(B+S)\right\|_{2}^{2}+\lambda_{b}\|B\|_{*}+\lambda_{s}\|S\|_{1} \\
\text { s.t. } \quad\left|\Theta_{(i, i)}\right|=1 \quad \forall i
\end{aligned}
$$

where $\lambda_{b}$ and $\lambda_{s}$ are regularization parameters. The first term enforces the data fidelity, the second term enforces the matrix to be low-rank and the third term enforces sparsity. It is not straightforward to solve this problem with current LRSD methods since it involves the data fidelity term. Therefore, we use variable splitting by introducing a variable $F$ such that $F=B+S$. The resulting constrained optimization problem 
takes the following form:

$$
\begin{aligned}
\widehat{F}, \widehat{B}, \widehat{S}, \widehat{\Theta}=\arg \min _{F, B, S, \Theta}\left\|g-H \Theta R^{*}(F)\right\|_{2}^{2}+\lambda_{b}\|B\|_{*}+\lambda_{s}\|S\|_{1} \\
\text { s.t } \quad F=B+S \\
\text { s.t. } \quad\left|\Theta_{(i, i)}\right|=1 \quad \forall i
\end{aligned}
$$

In the image domain, $S$ represents the image that contains sparse components, $B$ represents the approximately low-rank background image, and $F$ represents the composite image. The proposed method enforces the low-rank constraint to the patches of the image. This enforces that small regions of the background are correlated. The augmented Lagrangian form of this problem can be expressed as follows:

$$
\begin{aligned}
L(F, B, S, \Theta, Z)= & \left\|g-H \Theta R^{*}(F)\right\|_{2}^{2}+\lambda_{b}\|B\|_{*}+\lambda_{s}\|S\|_{1} \\
& +\langle Z, F-B-S\rangle+\frac{\beta}{2}\|F-B-S\|_{F}^{2} \\
& \text { s.t. } \quad\left|\Theta_{(i, i)}\right|=1 \quad \forall i
\end{aligned}
$$

where $Z$ is the Lagrange multiplier, and $\beta>0$ penalizes the violation of the constraint. We use alternating direction method of multipliers (ADMM) [17] for the solution of the problem. In particular, we introduced an auxiliary variable $F$ in order to solve for $B$ and $S$ separately. We minimize the problem over one variable while keeping the other variables fixed.

\section{Solution of the Optimization Problem}

There are five different variables to be solved for: $F, B, S, Z, \Theta$. For each iteration, each of these variables is solved for by keeping the other variables fixed by using ADMM. Below, we describe the process of updating each of these variables for a generic $(k+1)^{s t}$ iteration.

\section{Solution of the Sparse Matrix $S^{(k+1)}$}

This step solves for the sparse matrix $S$ while keeping the other variables fixed. Dropping the constant terms, the subproblem of interest takes the form of

$$
\begin{aligned}
S^{(k+1)}=\arg \min _{S} \lambda_{s}\|S\|_{1} & +\left\langle Z^{(k)}, F^{(k)}-B^{(k)}-S\right\rangle \\
& +\frac{\beta}{2}\left\|F^{(k)}-B^{(k)}-S\right\|_{F}^{2}
\end{aligned}
$$

This problem is equivalent to the well-known LASSO problem and can be solved via soft thresholding. The soft thresholding operator can be expressed as follows:

$$
\widetilde{C}_{\epsilon}(S)= \begin{cases}S-\epsilon & \text { if } S>\epsilon \\ S+\epsilon & \text { if } S<-\epsilon \\ 0 & \text { otherwise }\end{cases}
$$

and the solution of the problem is $S^{(k+1)}=\widetilde{C}_{\frac{\lambda_{s}}{\beta}}\left(F^{(k)}-B^{(k)}+\right.$ $\left.\frac{Z^{(k)}}{\beta}\right)$.

\section{Solution of the Low-rank Matrix $B^{(k+1)}$}

In this step, we update the low-rank matrix $B$. The subproblem for this step is

$$
\begin{aligned}
B^{(k+1)}=\arg \min _{B} \lambda_{b}\|B\|_{*} & +\left\langle Z^{(k)}, F^{(k)}-S^{(k+1)}-B\right\rangle \\
& +\frac{\beta}{2}\left\|F^{(k)}-S^{(k+1)}-B\right\|_{F}^{2}
\end{aligned}
$$

This subproblem involves nuclear norm minimization and its subgradient computation can be done through singular value

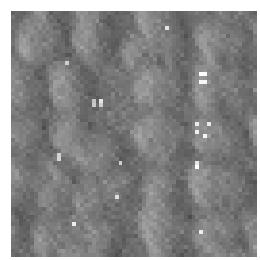

(a)

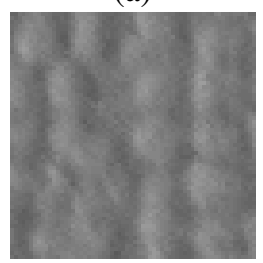

(d)

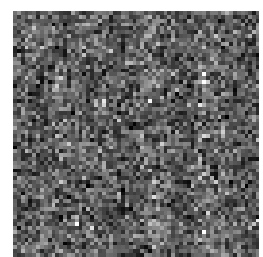

(b)

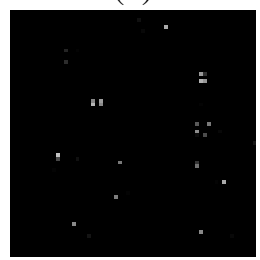

(e)

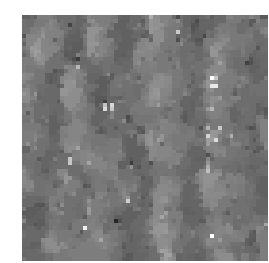

(c)

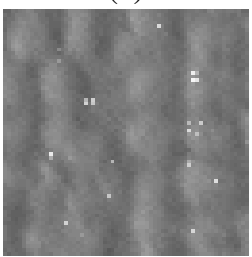

(f)
Fig. 1. Results of the synthetic scene experiment with $L=0.71$. (a) Original scene. (b) Conventional reconstruction MSE $=0.0310$. (c) Pointregion enhanced regularization MSE $=0.0015$. (d) The low-rank component produced by the proposed approach. (e) The sparse component produced by the proposed approach (f) The composite image MSE $=0.0008$.

thresholding [18]. In particular the soft thresholding operator is applied on the singular values of the matrix. The solution of this problem can be expressed as follows:

$$
S^{(k+1)}=U_{k} \widetilde{C}_{\frac{\lambda_{b}}{\beta}}\left(\Sigma_{k}\right) V_{k}^{T}
$$

where $U_{k} \Sigma_{k} V_{k}^{T}$ is the singular value decomposition of the matrix $F^{(k)}-S^{(k+1)}+\frac{Z^{(k)}}{\beta}$. Both singular value thresholding and soft thresholding are element-wise operations. Thus, these operations are computationally efficient except for the computation of the singular value decomposition.

\section{Solution of the Patch-based Matrix $F^{(k+1)}$}

In this step, we update the patch-based matrix $F$ for the following subproblem.

$$
\begin{aligned}
F^{(k+1)}=\arg \min _{F}\left\|g-H \Theta^{(k)} R^{*}(F)\right\|_{2}^{2} & +\left\langle Z^{(k)}, F-S^{(k+1)}-B^{(k+1)}\right\rangle \\
& +\frac{\beta}{2}\left\|F-S^{(k+1)}-B^{(k+1)}\right\|_{F(15)}^{2}
\end{aligned}
$$

This subproblem is quadratic and can be solved analytically. Taking the derivative with respect to $F$ and equating it to zero gives the following equation.

$$
\begin{aligned}
& \left(2\left(H \Theta^{(k)} R^{*}\right)^{H}\left(H \Theta^{(k)} R^{*}\right)+\beta I\right) F^{(k+1)} \\
& =\left(2\left(H \Theta^{(k)} R^{*}\right)^{H} g+\beta\left(B^{(k+1)}+S^{(k+1)}\right)-Z^{(k)}\right)
\end{aligned}
$$

We solve this problem with a few conjugate gradient steps.

\section{Solution of the Phase Matrix $\Theta^{(k+1)}$}

For this solution we introduce a vector $p$ consisting of the diagonal elements of the phase matrix $\Theta$. The corresponding subproblem is

$$
\widehat{p}=\arg \min _{p} \| g-\left.H \widetilde{M} p\right|_{2} ^{2}+\lambda_{p} \sum_{i=1}^{N}\left(\left|p_{i}\right|-1\right)^{2}
$$

where $\widetilde{M}$ is the diagonal matrix consists of the elements of $R^{*}\left(F^{(k+1)}\right)$ and $\lambda_{p}$ is Lagrange multiplier. This problem is similar to the phase update step involved in [9]. We solve this problem through a fixed point algorithm. 


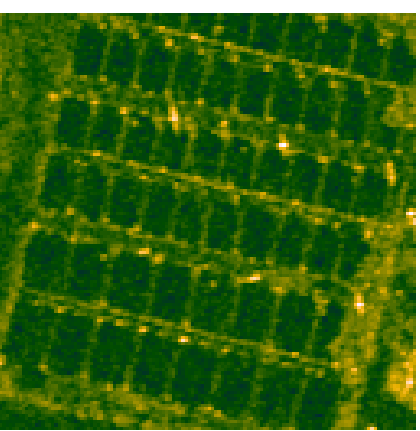

(a)

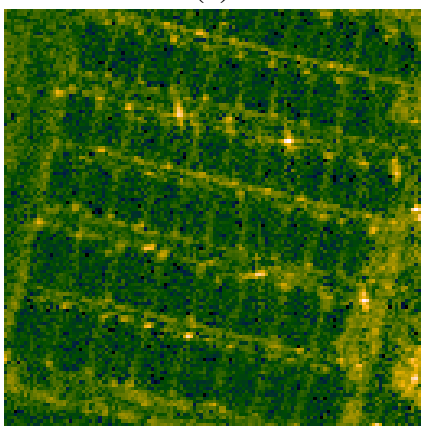

(c)

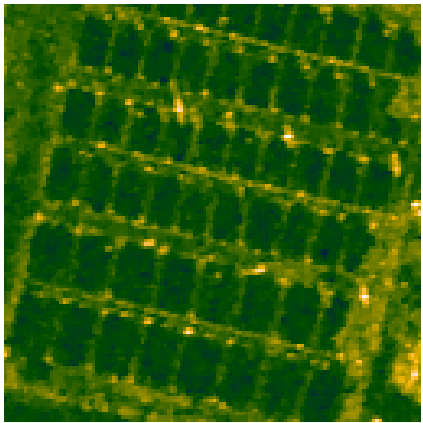

(e)

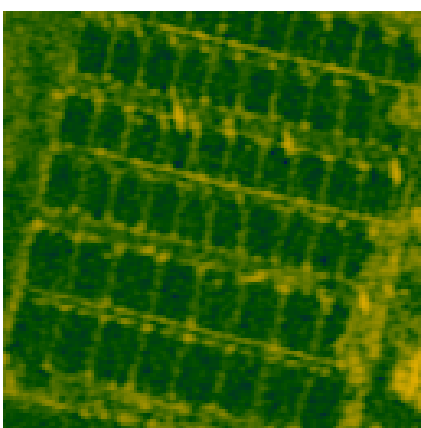

(b)

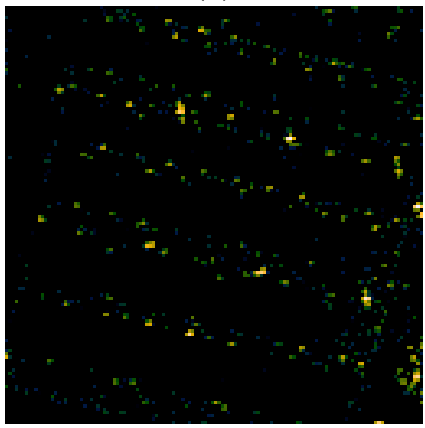

(d)

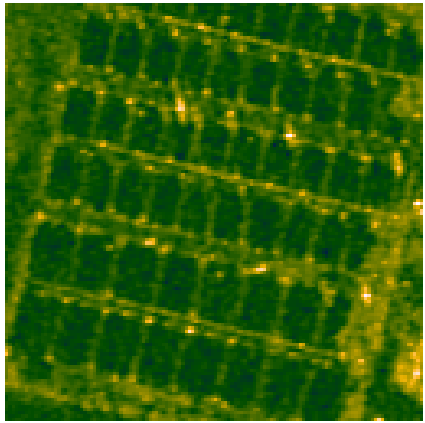

(f)

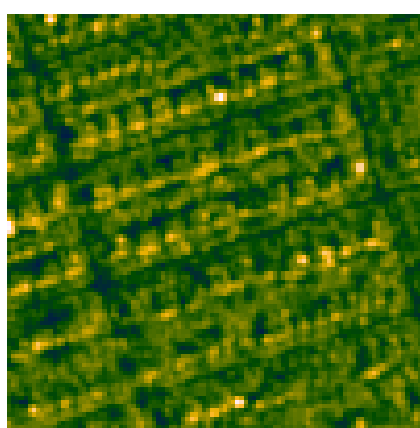

$(\mathrm{g})$

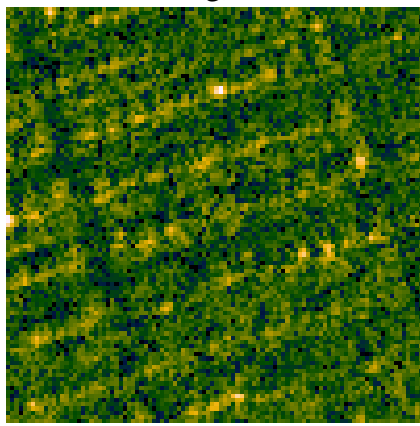

(i)

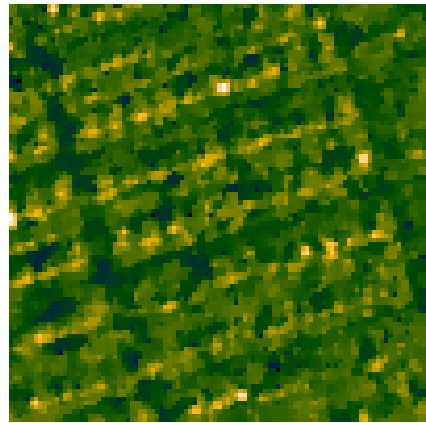

(k)

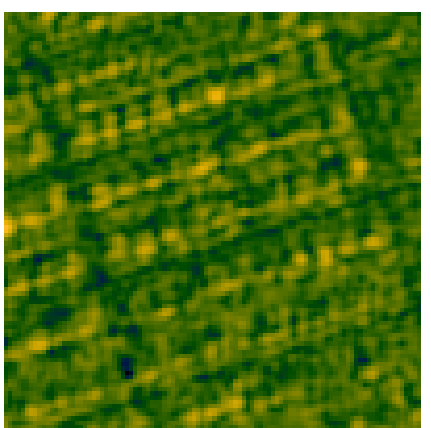

(h)

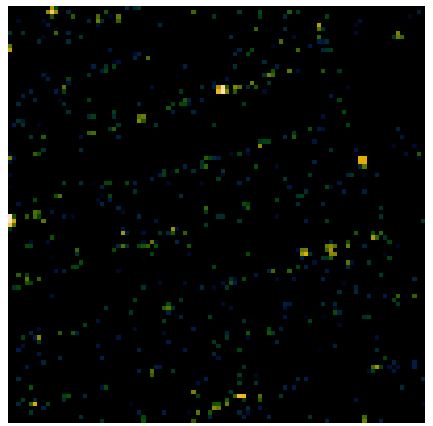

(j)

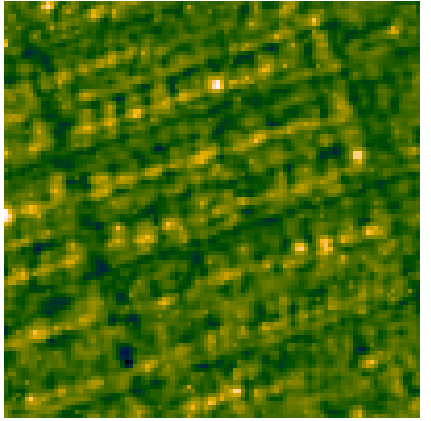

(1)

Fig. 2. Results of the real SAR scene experiments with $L=0.9$ (a-f) and 0.77 (g-1). First experiment: (a) Reference image conventionally reconstructed from full data. (c) Conventional reconstruction. (e) Point-region enhanced regularization. (b) The low-rank component produced by the proposed approach. (d) The sparse component produced by the proposed approach (f) The composite image produced by the proposed approach. Second experiment: (g) Reference image conventionally reconstructed from full data. (i) Conventional reconstruction. (k) Point-region enhanced regularization. (h) The low-rank component produced by the proposed approach. (j) The sparse component produced by the proposed approach (l) The composite image produced by the proposed approach.

\section{Lagrange Multiplier Update and Continuation}

We update the Lagrange multiplier $Z$ at each iteration with step size $\beta$. In particular, $Z$ is updated as follows:

$$
Z^{(k+1)}=Z^{(k)}+\beta\left(F^{(k+1)}-B^{(k+1)}-S^{(k+1)}\right)
$$

Note that when the value of $\beta$ is high, the ALM form of the optimization problem approaches the constrained version of the problem in (9). However, large value of $\beta$ leads to slow convergence of the algorithm. Thus, we use a continuation strategy for $\beta$. In particular, we start with small $\beta$ and at each iteration we increase $\beta$ slowly. These iterative steps are run until the convergence criterion $\frac{\left\||F|^{(k+1)}-|F|^{(k+1)}\right\|_{F}}{\left\||F|^{(k)}\right\|_{F}}<\delta_{x}$ is satisfied.

\section{EXPERIMENTAL RESULTS}

We present experimental results evaluating the performance of the proposed method using both synthetic scenes and real
SAR scenes from the TerraSAR-X [19] dataset. We provide quantitative results on the synthetic scene experiments. In the experiments we used a band-limited Fourier transform as our forward model $H$. We display the sparse part, the lowrank background part and the composite image produced by the proposed approach. We compare the performance of the proposed method with conventional reconstruction methods and point-region enhanced regularization method [8] with respect to mean squared error (MSE).

\section{A. Synthetic Scene Experiment}

We use a $64 \times 64$ synthetic scene for the first set of experiments. We compose a synthetically constructed sparse scene and a low-rank background scene. The composite scene is presented in Fig. 1(a). Note that, the composite image can be seen as man-made metallic scatterers on natural terrain. Uniformly distributed random phase between $[-\pi, \pi]$ is added to the composite image. We add Gaussian noise to the phase 
history data generated using a band-limited Fourier transform operator as mentioned above. We aim to reconstruct an estimate of the low-rank background, the sparse part and the composite image based on these phase histories. We present synthetic scene experiment results for $L=0.71$ in Fig. 1 where $L$ is the available data ratio. In particular $L=\frac{N_{a}}{N_{a}}$ where $N_{a}$ is the number of available data samples and $N_{d}$ is the number of phase history samples in full-bandwidth data. The proposed method provides an accurate composite image as compared to the other methods. Furthermore, it produces an accurate sparse part and a low-rank background for potential further interpretation. As an example, if the task of the SAR application is detection of these sparse parts, the sparse part from the proposed method can be used for such analysis. Point-region enhanced regularization provides comparable reconstruction results yet it suffers from artifacts and fails to provide an efficient reconstruction for the background of the image.

\section{B. Real SAR Scene Experiments}

We now present experimental results on real SAR scenes obtained from the TerraSAR-X dataset. In the first experiment we use SAR data that have been collected in a staring spotlight mode with $0.85 \mathrm{~m}$ range and $0.35 \mathrm{~m}$ azimuth resolution. We use $128 \times 128 \mathrm{SAR}$ data for the experiments. The test image contains a water treatment facility in Egypt (Fig. 2(a)). Note that this image contains repeating objects. For an appropriate selection of patch size, columns of the patch-based matrix will be similar to each other implying that the patch-based matrix is low-rank. Therefore, the patch-based low-rank component in our approach enforces that small regions in the image are similar. Thus, this test image is a good candidate for the LRSD framework. Available data ratio is set to $L=0.9$ for this experiment. Reconstruction results are shown in Fig. 2(af). The proposed method appears to effectively decompose the background and the sparse part of the SAR scene and it provides a similar reconstruction result with point-region enhanced regularization. The sparse part contains the point scatterers around the repeating objects and the background part enhances these repeating objects. Each of these two components/layers could be useful for scene analysis and interpretation.

In the second experiment, we have used SAR data collected in a spotlight mode with $3.75 \mathrm{~m}$ range and $3.69 \mathrm{~m}$ azimuth resolutions. Image size is $128 \times 128$. The test image covers a region in Toronto containing roads and small houses (Fig. $2(\mathrm{~g})$ ). Note that, these structures exhibit similarity as in the case for the first experiment hence low-rank background can be enforced on these structures. We consider an available data ratio of $L=0.77$ for the second experiment. Reconstruction results are shown in Fig. 2(g-l). The proposed method captures the sparse scatterers in the sparse part and the low-rank structures are well preserved in the low-rank background image as compared to the point-region enhanced regularization and conventional methods. In particular, note that, the background part preserved the road and small repeating objects in the scene.

\section{CONCLUSiON}

We have proposed a framework for SAR image formation based on a low-rank and sparse decomposition of the scene to be imaged. Most of the imaging applications involving SAR images require background subtraction or segmentation for better interpretation of the image. Thus, we have proposed a SAR image reconstruction framework that separates background from sparse regions in the process of reconstructing the SAR image. Such an approach integrated into image formation may offer benefits over a post-processing approach aimed at such image decomposition. We have shown that this approach provides accurate reconstruction performance as compared to existing reconstruction methods. Moreover, separating background from sparse regions may facilitate further SAR image analysis. In our work we enforce sparsity on the SAR image directly. In future work sparsity can be imposed in a different domain through the use of sparsifying transforms or a representation dictionary. Moreover, the proposed LRSDbased SAR image reconstruction framework can potentially be used in different SAR imaging scenarios such as wide-angle and moving target imaging.

\section{REFERENCES}

[1] J.-L. Starck, M. Elad, D.L. Donoho, "Image decomposition via the combination of sparse representations and a variational approach," IEEE Transactions on Image Processing, vol.14, no.10, pp.1570,1582, Oct. 2005.

[2] E. J. Candès, X. Li, Y. Ma, and J. Wright, "Robust principal component analysis?" J. ACM, vol. 58, no. 3, pp. 11:1-11:37, Jun. 2011.

[3] C.-F. Chen, C.-P. Wei, and Y.-C. Wang, "Low-rank matrix recovery with structural incoherence for robust face recognition," in Computer Vision and Pattern Recognition (CVPR), 2012 IEEE Conference on, June 2012, pp. 2618-2625.

[4] T. B. C. Guyon and E. Zahzah, Robust Principal Component Analysis for Background Subtraction: Systematic Evaluation and Comparative Analysis. INTECH, 2012 .

[5] S. Lingala, Y. Hu, E. DiBella, and M. Jacob, "Accelerated dynamic MRI exploiting sparsity and low-rank structure: k-t SLR," IEEE Transactions on Medical Imaging, vol. 30, no. 5, pp. 1042-1054, May 2011.

[6] W. Carrara, R. Majewski, and R. Goodman, Spotlight Synthetic Aperture Radar: Signal Processing Algorithms. Artech House, 1995.

[7] D. C. Munson, Jr., J. D. O'Brien, and W. K. Jenkins, "A tomographic formulation of spotlight-mode synthetic aperture radar," Proc. IEEE, vol. PROC-71, pp. 917-925, 1983

[8] M. Çetin and W. C. Karl, "Feature-enhanced synthetic aperture radar image formation based on nonquadratic regularization," IEEE Trans. Image Processing, pp. 623-631, 2001.

[9] S. Samadi, M. Cetin, and M. Masnadi-Shirazi, "Sparse representation-based synthetic aperture radar imaging," IET Radar, Sonar and Navigation, vol. 5, no. 2, pp. 182-193, 2011.

[10] E. Candès and B. Recht, "Exact matrix completion via convex optimization," Foundations of Computational Mathematics, vol. 9, no. 6, pp. 717-772, 2009.

[11] B. Recht, M. Fazel, and P. Parrilo, "Guaranteed minimum-rank solutions of linear matrix equations via nuclear norm minimization," SIAM Review, vol. 52, no. 3, pp. 471-501, 2010.

[12] Z. Lin, M. Chen, and Y. Ma, "The Augmented Lagrange Multiplier Method for Exact Recovery of Corrupted Low-Rank Matrices," ArXiv e-prints, Sep. 2010.

[13] J. X. Yuan, "Sparse and Low-Rank Matrix Decomposition Via Alternating Direction Methods," Optimization Online, 2009.

[14] M. Dao, L. H. Nguyen, and T. D. Tran, "Temporal rate up-conversion of synthetic aperture radar via low-rank matrix recovery," in IEEE International Conference on Image Processing, ICIP 2013, Melbourne, Australia, September 15-18, 2013. IEEE, 2013, pp. 2358-2362.

[15] D. Yang, G. Liao, S. Zhu, X. Yang, and X. Zhang, "Sar imaging with undersampled data via matrix completion," IEEE Geoscience and Remote Sensing Letters, vol. 11, no. 9, pp. 1539-1543, Sept 2014.

[16] C. Gao, D. Meng, Y. Yang, Y. Wang, X. Zhou, and A. Hauptmann, "Infrared patchimage model for small target detection in a single image," IEEE Transactions on Image Processing, vol. 22, no. 12, pp. 4996-5009, Dec 2013.

[17] S. Boyd, N. Parikh, E. Chu, B. Peleato, and J. Eckstein, "Distributed optimization and statistical learning via the alternating direction method of multipliers," Found. Trends Mach. Learn., vol. 3, no. 1, pp. 1-122, Jan. 2011.

[18] J.-F. Cai, E. J. Candes, and Z. Shen, "A Singular Value Thresholding Algorithm for Matrix Completion," ArXiv e-prints, Oct. 2008.

[19] Astrium TerraSAR-X sample imagery. [Online] Available:http://www.astriumgeo.com/en/23-sample-imagery 